\title{
A new window sheds light on lung tumor metastasis
}

The cellular mechanisms of tumor invasion and expansion during metastasis in the lungs have been a black box for decades, understood only by inference using relatively low-resolution imaging techniques. But thanks to some moxie and a T32 training grant at Montefiore's Department of Surgery, the precise details in mouse models are beginning to reveal themselves, as demonstrated in a recent Nature Method's paper (Nat. Methods 15, 73-80; 2018).

David Entenberg is a physicist and engineer who develops novel instrumentation for microscopy at the biophotonics center at the Einstein College of Medicine, where he has been collaborating with cancer researcher John Condeelis since 2006. A couple of years ago the two teamed up to apply a vacuum lung imaging window to study tumor biology in mouse lungs using intravital imaging. Although successful, the group had pushed the surgical technique to its limits and were frustrated by the small experimental timeframe allowed by the method, which requires a highly invasive surgical procedure in terminally anesthetized animals. To capture cellular mechanisms of events like tumor metastasis, a common occurrence in the lungs and a primary scientific aim of the Condeelis lab, the team needed to be able to image lungs in mice over a timespan of weeks, not the 12 hours typical of terminally anesthetized preps.

"So I tossed out the idea, why don't we try to make a window where the mouse could survive and wake-up," says Entenberg, "and everybody basically said, there's no way that's going to be possible."

This happened to coincide with the Montefiore Medical Center (the university hospital of Einstein) receiving a T32 training grant from the NIH to specifically bring surgeons into the lab to get some science

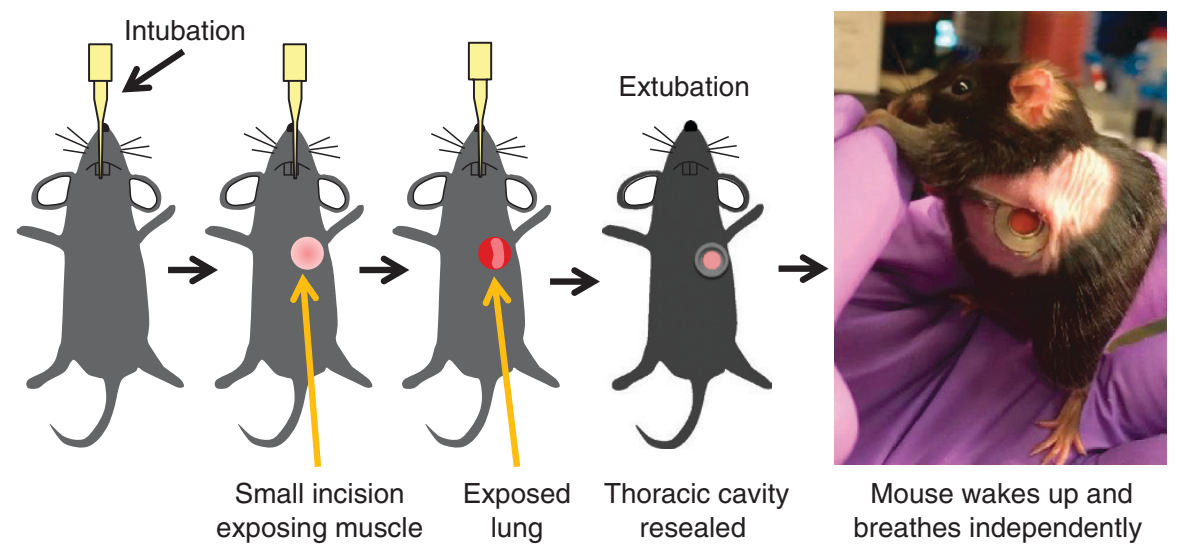

Overview of surgical procedure and example recovered mouse after implantation. Image adapted from Nat. Methods 15, 73-80 (2018).

experience. Sonia Voiculescu, a surgical resident at Einstein, joined the Condeelis lab, and teamed up with Entenberg to provide the surgical expertise and perspective necessary to turn Entenberg's crazy idea into a reality. The two set out to develop a novel imaging window in the mouse lungs that would enable the animals to wake up and fully recover after surgery, allowing for serial microscopy in the same lungs at cellular resolution. "I had developed the engineering design for the window," says Entenberg, "and Sonia came in with her surgical skills and together we started pushing forward what we call surgical engineering." Rather than developing a robot or new material for the operating room, which, according to Entenberg, is the typical province of surgical engineering, "we flipped the concept on its head and brought the knowledge and skills that surgeons have in the OR and combined it with our engineering to develop a novel protocol."

The protocol, described in detail in their paper, uses a small glass window implanted over the lungs for high-resolution cellular imaging. Because the procedure completely reseals the thoracic cavity after implantation, the mice can recover and breath comfortably on their own, ready for reimaging over the course of weeks. Using mouse models of lung tumor metastasis, the team was able to image individual tumor cells invading and expanding in the murine lungs, an accomplishment that opens up new avenues for testing long-held assumptions about tumor metastasis in the lungs.

"Our interests going forward are to focus in on the lung and focus in on metastasis," says Entenberg. "Before it was a black box and now we have the ability to directly visualize it over days and days and see what actually happens. We have the ability to look at that physiological process over time and test conclusions that have been based upon old experimental metastasis assays, and find out which ones hold up under more realistic biological conditions." Dustin M. Graham 